\title{
Los espacios transfronterizos europeos: ¡un objeto geográfico de difícil definición? Una aproximación desde la perspectiva de los soft spaces*
}

\section{David Giband}

Université de Perpignan Via Domitia. Département Géographie et Aménagement du Territoire

giband@univ-perp.fr

Joan Vicente Rufí

Universitat de Girona. Departamento de Geografía

joan.vicente@udg.edu

\section{Resumen}

El texto propone una visión de los espacios transfronterizos desde la perspectiva de los soft spaces - los espacios blandos- con relación al planeamiento territorial. Con ella se consigue una interpretación mucho más ajustada de la realidad de las áreas transfronterizas, así como de sus dinámicas. Por otra parte, el artículo hace un repaso a lo que ha sido la cooperación transfronteriza en Europa desde sus orígenes hasta llegar al presente. Partiendo de una coincidencia con otros autores en la definición de fases, se acaba proponiendo que la última, iniciada en 2008, se caracterizaría por la tensión entre las fuerzas que reclaman una recuperación de la frontera y la soberanía de los estados y las que, contrariamente, propugnan recuperar la idea de integración europea perdida en la última década. Es decir, precisamente una tensión entre los soft spaces transfronterizos y un rebordering incipiente.

Palabras clave: Unión Europea; INTERREG; cooperación transfronteriza; soft spaces; rebordering

* Este texto forma parte de la investigación La cooperación transfronteriza en Europa, una geopolitica de escala local. Análisis en cinco países europeos de buenas prácticas para la integración y el desarrollo global (TRANSBORDEURCOP), financiado por el Ministerio de Economía y Ciencia. CSO2015-67124-R. 
Resum. Els espais transfronterers europeus: un objecte geogràfic de difícil definició? Una aproximació des de la perspectiva dels soft spaces

El text proposa una visió dels espais transfronterers des de la perspectiva dels soft spaces - espais tous - en relació amb el planejament territorial. Amb aquesta s'aconsegueix una interpretació molt més ajustada del que són en realitat les àrees transfrontereres així com de les dinàmiques que s'hi produeixen. D'altra banda, l'article fa un repàs del que ha estat la cooperació transfronterera a Europa fins arribar al present. Partint de la coincidència amb altres autors en la definició de fases, s'acaba proposant que l'última, iniciada el 2008, es caracteritzaria per la tensió entre les forces que reclamen una recuperació de la frontera i la sobirania dels estats i les que, contràriament, propugnen recuperar la idea d'integració europea perduda en la darrera dècada. És a dir, precisament una tensió entre els soft spaces transfronterers i un rebordering incipient.

Paraules clau: Unió Europea; INTERREG; cooperació transfronterera; soft spaces; rebordering

Résumé. Les espaces transfrontaliers européens: un objet géographique difficile à définir? Une approche du point de vue des soft spaces

Le texte propose une vision des espaces transfrontaliers du point de vue des espaces mous - soft spaces - en relation avec l'aménagement du territoire. Cette vision nous permet de parvenir à une interprétation beaucoup plus fine de ce que sont réellement les zones transfrontalières, ainsi que de la dynamique qui s'y produit. Par ailleurs, l'article passe en revue le rôle de la coopération transfrontalière en Europe jusqu'à présent. Partant de la coïncidence avec d'autres auteurs dans la définition des termes, il finit par proposer une dernière définition, initiée en 2008, qui serait caractérisée par la tension entre les forces qui exigent une récupération de la frontière et la souveraineté des États et celles qui, au contraire, suggèrent de récupérer l'idée d'une intégration européenne perdue au cours de la dernière décennie, c'est-à-dire, précisément, une tension entre les soft spaces transfrontaliers et un rebordering naissant.

Mots-clés: Union Européenne; INTERREG; coopération transfrontalière; soft spaces; rebordering

Abstract. European cross-border spaces: A difficult to define geographic object? An approach from the perspective of soft spaces

This text proposes a vision of cross-border spaces from the perspective of soft spaces in relation to territorial planning. In doing so, we aim to achieve a much more realistic interpretation of cross-border areas, as well as the dynamics that occur in them. The article also examines cross-border cooperation in Europe from its origins to the present. Starting with a definition of phases coinciding with other authors, the article concludes with the proposal of a last one initiated in 2008, which is characterized by the tension between forces that call for the recovery of the border and the sovereignty of the states and those who, on the contrary, advocate recovering the idea of European integration lost in the last decade; that is, a tension between the transboundary soft spaces and an emerging rebordering.

Keywords: European Union; INTERREG; cross-border cooperation; soft spaces; rebordering 


\section{Sumario}

1. Introducción

2. Espacios transfronterizos: espacios que necesitan definición

3. Los espacios transfronterizos y la cooperación transfronteriza a lo largo del proceso de construcción de la Unión Europea
4. ¿De la cohesión territorial a los espacios blandos?

5. Conclusión: los espacios transfronterizos como espacios blandos y la crisis de la Unión Europea: entre la desfronterización y la refronterización Referencias bibliográficas

\section{Introducción}

Desde que el Convenio de Madrid (1980), promovido por el Consejo de Europa, explicitó el interés de los estados por la cooperación transfronteriza, esta se ha convertido en uno de los principales objetivos de la política de cohesión territorial de la Unión Europea (UE). El tema ha sido objeto de abundante literatura científica (Perkmann, 2003; Scott, 2005; Harguindéguy y Hayward, 2012). Esta abundancia ha servido para poner de relieve la gran diversidad de formas, escalas y procesos de cooperación territorial transfronteriza; diversidad que más que simplificar dificulta la definición de áreas transfronterizas (Hamez, 2013). De hecho, detrás del propio término de espacio transfronterizo se encuentran realidades geográficas complejas y múltiples que operan a diferentes escalas — desde la local (o de proximidad) hasta la regional-, generando, a su vez, redes más o menos estructurantes y movilizando actores de toda índole.

Las áreas fronterizas, a pesar de incluir a casi el 30\% de la población europea en su acepción estrictamente administrativa y que se definan como áreas estratégicas de la UE, todavía parecen difíciles de delimitar y comprender. El propósito de este artículo es, en primer lugar, interrogarse sobre la naturaleza geográfica de los espacios transfronterizos y sus dinámicas. Estos, a menudo sin territorio preestablecido o con geografía variable, son espacios en los que sus lógicas de funcionamiento dependen de procesos que se combinan en escalas múltiples. Aparecen como espacios en constante evolución, cuya construcción sigue ligada fundamentalmente a las modalidades y el ritmo de la construcción europea. Es decir, la realidad espacial de las áreas transfronterizas aparece hoy en Europa como algo inseparable de la política de cooperación iniciada tímidamente en los años cincuenta por los actores institucionales primeramente locales y regionales y luego estatales y, finalmente, a principios de los años ochenta, bajo la política de integración hacia la futura UE.

Por otro lado, respecto a la evolución de la cooperación transfronteriza, Oliveras, Durà y Perkmann (2010) identifican tres etapas, y anuncian una cuarta en base a las nuevas directrices de la política de cohesión territorial europea formalizada en 2007 a través del programa Europa 2020. Una última etapa que significaría la transición de una política de cooperación transfronteriza a 
una política de cooperación territorial definida como uno de los objetivos de la política regional de la UE. Detrás de este cambio semántico debemos leer, tras la reforma de 2007 de los fondos estructurales, la implementación de una política de cooperación territorial caracterizada por la competencia, la búsqueda del atractivo y la adaptación de los territorios transfronterizos al mercado y a las dinámicas de la globalización.

Confrontados con los tres objetivos de la reforma de 2007 (convergencia, competitividad e innovación y cooperación territorial), argumentaremos que los espacios de cooperación transfronteriza se configuran como espacios polimórficos y en construcción permanente similares a los que Andreas Faludi (2013) califica como "espacios blandos» (soft spaces): espacios fluidos con configuraciones cambiantes, límites inciertos y borrosos. Esta aparente fluidez — y lo que puede describirse como incertidumbre espacial— no será el resultado de la mera superposición institucional y administrativa propia de los dispositivos de la UE, sino que también puede aparecer como una respuesta estructurada a la reconfiguración de la política espacial europea como consecuencia de un proceso de reajuste escalar — un rescaling process(Brenner, 1999) más amplio.

Partiendo de estas bases, el presente artículo se estructura en tres apartados — sin contar la introducción y las conclusiones-, el primero de los cuales busca resaltar las dificultades para definir con precisión los espacios transfronterizos — analizando en particular el registro semántico asociado a ellos-. A continuación se aborda la vinculación entre estos espacios y su definición con la evolución de las políticas de cooperación europea y sus vaivenes, con especial atención al período que se inicia en 2007, en el que coinciden nuevas directrices políticas y la crisis económica y geopolítica global. En el tercer apartado mostraremos que los espacios internos transfronterizos de la UE se constituyen como espacios blandos y las características asociadas a ellos: sin territorio real prefijado, con una geografía variable y diversas configuraciones. Como ejemplo veremos brevemente como la cooperación transfronteriza entre España y Francia responde a esta configuración imprecisa del espacio, partiendo de los datos provenientes de INTERREG III y IV.

Las reflexiones de estos tres apartados harán emerger la situación paradójica de unos espacios transfronterizos articulados local/regionalmente como soft spaces y una realidad europea que en la última década ve cómo las fronteras y el concepto de soberanía estatal recuperan un protagonismo por muy diversas vías y motivos, contrario en buena medida al «espíritu» que alimentó el proceso de integración desde mediados de los años ochenta.

\section{Espacios transfronterizos: espacios que necesitan definición}

La cuestión de las fronteras internas y las zonas fronterizas de Europa alimenta una abundante literatura científica, pero sin llegar a definir con precisión el término espacio o territorio transfronterizo. Como han señalado Kaplan y Hakli (2002), la variedad de términos y los enfoques utilizados para describir el 
carácter transfronterizo dificultan la conceptualización o permiten muy diversas conceptualizaciones, más todavía si se tienen en cuenta variedades idiomáticas, por ejemplo, entre lenguas latinas y anglosajonas (Berzi, 2017b).

\subsection{Fronteras, un objeto complejo}

Para abordar el concepto de espacio transfronterizo, primero es necesario volver al cambio de paradigma en el concepto de fronteras. Nuestra ambición no es proporcionar una revisión exhaustiva de la literatura sobre la frontera y sus enfoques en Geografía, tema que no podríamos agotar en un artículo, sino modestamente anotar los cambios recientes de esta noción con respecto a nuestro objeto de estudio: los espacios transfronterizos. Como ha señalado Brunet-Jailly (2005), no se puede entender los espacios transfronterizos sin cuestionar, en el contexto de la globalización y de reestructuraciones territoriales, la noción de frontera a través de una mirada compleja: de las fuerzas del mercado y los flujos de intercambio, de las acciones políticas que intervienen alrededor de las fronteras, de las comunidades políticas que se forman en ellas y de sus especificidades culturales. Tantos cambios que hacen de los espacios afectados por las fronteras realidades más complejas y diversas. Según Herzog y Sohn (2015): «Mostrándose como dinámicas y variables, las fronteras han sido interpretadas no como una línea que separa simplemente dos estados y que tiene necesidad de ser defendida sino como un medio extremadamente complejo que soporta procesos de fronterización» (p. 2).

Comprender las fronteras de hoy en día es interpretar los procesos sociales que alimentan dos dinámicas opuestas: desfronterización y refronterización. La primera sugiere la obertura, el intercambio y la hibridación, mientras que la segunda remite a prácticas de control, protección y diferenciación. Estos dos movimientos se oponen e interactúan mutuamente. Alimentan dinámicas funcionales (construcción de espacios institucionales), estructurales (construcción social) y simbólicas (nueva identidad espacial).

Asumiendo pues esta cuestión previa, como se sabe son múltiples los factores geopolíticos y geoeconómicos que conducen al debilitamiento de los estados nacionales y de su soberanía, particularmente en Europa, donde el proceso de construcción europea en la década de 1980 se basa en un redimensionamiento de esta entidad y sus valores (Agnew, 1994; Castells, 1998; Brenner, 1999; Paasi, 2009). En base a ello, las fronteras, anteriormente los márgenes y las periferias de Europa, están destinadas a convertirse en laboratorios de integración europea y en piedra angular de la política de cohesión territorial. A partir de este discurso preformativo, se pone en marcha un imaginario político sobre la cohesión territorial que no produce efectos patentes en materia de reducción de las diferencias de desarrollo en favor de las regiones transfronterizas. Se producen complejos movimientos debidos a la «desfronterización», «refronterización» (debordering y rebordering) en base a la desactivación parcial de las fronteras internas de la UE y a las cuestiones geopolíticas estructurales 
(ampliaciones de la UE, fortalecimiento de las fronteras perimetrales, creación de límites intracomunitarios bajo el área de Schengen), lo que lleva a la transformación de los límites de los objetos geográficos simples que ciñen dos soberanías territoriales en «un objeto complejo que es un lugar de relaciones paradójicas» (Piermay, 2009).

Lejos de desaparecer, las fronteras se han movido, multiplicado y resignificado. En la UE conservan parcialmente su carácter discriminatorio (entre los sistemas legales estatales), pero también abren la expectativa para una gran cantidad de actores como un recurso que alimenta oportunidades de desarrollo (Berzi, 2017b). Las fronteras internas de la UE, valoradas como recursos, han transformado la relación entre las áreas fronterizas (Sohn, 2014a). Estas se limitaron durante mucho tiempo al papel de los espacios de barrera que fijan los flujos (los spaces of flows), después se convirtieron en espacios de lugares (los spaces of places) (Blatter, 2004), fuentes de un sentido nuevo (para construir Europa) y de recursos (financieros, simbólicos e institucionales) para las áreas implicadas. Estos espacios se conforman, al ritmo de la construcción europea, como "territorio del proyecto" que depende de la capacidad de los actores locales para establecer una cooperación mutua, una fuente de innovación institucional y territorial (Sohn, 2014b).

Markus Perkmann (2005) ha demostrado con el ejemplo de la Eurorregión entre los Países Bajos y Alemania cómo los actores regionales y estatales construyen un área de cooperación transfronteriza a escala amplia a través de políticas para establecer la gobernanza multinivel y de la capacidad de definir los contornos de una unión estratégica en esta área regional. La implementación de un área regional transfronteriza se basa en la capacidad de construir nuevas escalas territoriales, refiriendo la dinámica espacial transfronteriza a procesos de «reescalamiento» (rescaling), (Brenner, 1999). La evolución del estatus de las áreas fronterizas, pasando de la función de límites a la de lugares de producción de cooperación, pone ante la cuestión de si surgen nuevas relaciones con el territorio y de la capacidad de estas cooperaciones para producir espacios realmente transfronterizos.

\subsection{Una triple dificultad para definir espacios transfronterizos}

Muchos autores han destacado la polisemia y el polimorfismo de los espacios transfronterizos (Hamez, 2013). La dificultad para identificar la naturaleza de los espacios transfronterizos es a la vez ontológica y epistemológica. Así, los términos que se utilizan para representar la dimensión espacial de la frontera son múltiples: espacio, territorio, región, red... Su uso a menudo indiferenciado fomenta una confusión entre la naturaleza y las escalas transfronterizas; un desenfoque que supone una triple dificultad cuando se trata de apreciar la naturaleza geográfica del hecho transfronterizo: semántico, contextual y conceptual (Hamez, 2013). Semántico en primer lugar, porque las palabras para describir el cruce de fronteras son numerosas y llevan a representaciones a veces divergentes. La misma palabra transfronteriza plantea preguntas. Como 
recogen Amilhat-Szary y Fourny (2006) del sociólogo Paul Blanquart, detrás del nombre genérico de transfronterizo lo que reside es la diferencia entre lo que es el trans-y el inter-fronterizo. El prefijo trans-se refiere a la idea de cruzar el borde, mientras que el de inter-se refiere a la articulación en red y a la conexión. Lo transfronterizo es una lógica de contigüidad, continuidad espacial y proximidad posible gracias al cruce de la frontera hacia un vecino. Lo interfronterizo se refiere a una lógica de espacios interconectados que hace posible la conexión en un contexto marcado por discontinuidades espaciales. El uso frecuente de ambos términos genera confusión, y el adjetivo transfronterizo se refiere indiferentemente a ambas categorías, especialmente cuando el análisis se centra en la escala regional, que abarca estas dos dimensiones de «inter»y «trans».

Desde el punto de vista contextual se refiere a varias realidades, y a lo que Hamez (2013) describe como "unicidad» de cada espacio transfronterizo que se debe a su historia, su particular identidad geográfica, económica y de procesos sociales particulares (Bauder, 2011). La realidad transfronteriza es, en efecto, muy diversa: espacio (de flujos, de intercambios), territorio (administrativo, institucional) y red de actores. Conceptualmente, el aparato teórico que hace posible traducir la complejidad de los procesos a la práctica sigue siendo particularmente difícil de establecer (Brunet-Jailly, 2005). Recordemos, siguiendo Hamez (2013), que la palabra transfronterizo apareció por primera vez en el Convenio de Madrid de 1980, relativa a la cooperación transfronteriza entre las autoridades locales y que sustituye a la de interfronteriza, limitada a la cooperación internacional. La adopción misma del término transfronterizo para las dos lenguas oficiales del Convenio genera dudas; la elección de la traducción del término francés transfrontalier al inglés (transfrontier), mientras que la palabra de uso común es cross-border. Estos embrollos semánticos denotan diversas visiones del hecho. El adjetivo transfronterizo tiene así dos significados: el de un cruce de frontera y el de un principio superior que se impone a los actores preexistentes (principio de subsidiariedad). Sirve para designar las nuevas prácticas y acciones de una plétora de actores locales, a menudo inéditos en la construcción europea (autoridades locales, empresas, universidades, asociaciones, etc.). Conlleva fuertes representaciones: la de la abolición de las fronteras, la de un interior europeo abierto, la de nuevas escalas de regulación económica, social y política... Sinónimo de integración europea, simboliza el surgimiento de nuevas escalas institucionales (primero las eurorregiones, luego las agrupaciones europeas de cooperación territorial -AECT-).

Refleja, en términos más generales, el deseo de territorializar una realidad económica, social y cultural que todavía está por construir o que permanece latente y que, sin embargo, parece muy alejada de la vida cotidiana de millones de europeos. Si Sohn y Stambolic (2015) definen como transfronterizos los lugares donde el desarrollo económico y social se ve influenciado por la proximidad de la frontera, entonces se plantea la cuestión de la naturaleza geográfica del objeto frontera: ¿̨el espacio, el territorio, la red, la región, el lugar? 


\subsection{Los espacios transfronterizos: una geografia variable}

En la aproximación a los espacios transfronterizos se confrontan dos tradiciones de investigación. Por un lado, el trabajo en el campo de la geografía regional y de la geografía política ponen de relieve la noción de región transfronteriza (Crossborder region, CBR). Lo que se ha descrito como el enfoque de la cooperación transfronteriza (CBCA) considera «lo transfronterizo" como una construcción institucional a escala regional y local, cuyo objetivo es posicionar las áreas periféricas de la UE, definidas como posibles «espacios activos», en el centro de la construcción territorial europea. Estos trabajos se basan en una definición funcional de las regiones transfronterizas que vinculan su existencia a dos dimensiones: una dimensión escalar (los espacios transfronterizos se abordan a veces a escala microlocal; a veces, a escala macrorregional) y una dimensión institucional (la de espacios de cooperación más o menos instituidos) (Oliveras, Durà, Perkmann, 2010). El espacio transfronterizo se valora desde la perspectiva de la construcción regional, que la UE define como «regiones potenciales». Siguiendo a Perkmann (2003), estos trabajos que se interesan por lo transfronterizo desde una perspectiva macrorregional comparten el regionalismo de las áreas fronterizas, basándose en la presunta presencia de una realidad transfronteriza atrapada en un proceso de reajuste escalar (rescaling). Perkmann (2003) llevó a cabo una definición de los elementos conceptuales y un ensayo de tipología de las regiones transfronterizas. Según él, entran en juego tres variables: 1) la escala geográfica que depende del nivel de acción territorial de la cooperación, 2) la naturaleza jurídica de la cooperación y su intensidad, y 3) el tipo de actores involucrados. El resultado es una tipología de regiones fronterizas en cuatro grupos: regiones fronterizas de microcooperación integrada, macrocooperación integrada, microcooperación emergente y macrocooperación emergente.

Por otro lado, las obras relacionadas con la geografía social y cultural insisten en la dimensión identitaria transmitida por la frontera (van Houtum, 2010; Berzi, 2017a), llegando incluso a evocar su dimensión simbólicocognitiva (Gualini, 2003). Van Houtum, a diferencia de un enfoque de los flujos de la geografía económica (flow approach), que considera el espacio transfronterizo como un espacio abstracto de flujos e intercambios y a diferencia de los partidarios del CBCA, propone un enfoque de las personas (people approach). Esto se refiere a las interacciones que una frontera puede generar para las comunidades locales y lleva el foco del análisis a nivel individual. Lo transfronterizo toma plenamente el sentido de territorio (y no únicamente el de espacio o región) en el sentido de que está institucionalmente construido y social, cultural e identitariamente apropiado por los actores locales. El territorio transfronterizo se considera social y culturalmente dotado a partir de legados culturales, sociales, de identidad y lingüísticos que existían previamente al establecimiento de la frontera. Esta propuesta se une a trabajos sobre paisajes fronterizos — border landscapes—, que llegan incluso a reconocer 
la existencia de una «mentalidad fronteriza» (Rumley y Minghi, 1991). En Francia, los trabajos de Amilhat-Szary y Fourny (2006) refieren el análisis de los espacios transfronterizos a su territorialidad, inscribiéndola en una perspectiva cultural y antropológica más marcada. Otros autores, en la tradición de la Escuela Italiana de Geografía, defienden un enfoque territorial de la frontera. Según Berzi (2017b), el proceso de integración territorial de la UE representa un marco que no solo estimula la cooperación transfronteriza, sino que también reactiva el viejo capital relacional que está en la raíz de la gobernanza transfronteriza. Con la cooperación transfronteriza, hay un nuevo ciclo de reterritorialización que crea nuevas formas de capital territorial transfronterizo. Dando el ejemplo de la construcción del hospital transfronterizo de Puigcerdà entre Francia y España, Berzi destaca la reactivación de un antiguo capital relacional en ambos lados de la frontera, base de una dinámica territorial colectiva, inscribiéndose en la trayectoria histórica de esta región que cuenta con una fuerte identidad cultural.

En todo caso, independientemente de las escuelas de pensamiento, surge del análisis lo transfronterizo (espacio, territorio o región), definido por su carácter de proceso (Evrard, 2013). Este proceso toma cuerpo dentro de la realidad social y económica, pero también institucional. Se construye a partir de la herencia de las prácticas sociales, de los intercambios, de los flujos y de las representaciones comunes provenientes de un lado y otro de la frontera. Como resultado, lo transfronterizo es una estructura socioespacial que a veces se institucionaliza y/o a veces se consolida social y culturalmente. Puede existir en la territorialidad de la acción transfronteriza, como construcción que es política, económica, social o identitaria. Cualquiera que sea la base, se trata de áreas con geografía variable cuya dimensión espacial, la escala de referencia, depende en buena parte de las orientaciones definidas por los fondos estructurales europeos (INTERREG). Así, INTERREG A define la idea de proximidad; $\mathrm{B}$, de continuidad, y $\mathrm{C}$, de red (tabla 1), y es esto lo que a menudo les da a estos espacios transfronterizos la forma de estructuras enmarañadas y entrelazadas. Esta sobreposición de referencias geográficas y espaciales (de la proximidad local a la red) define, sin embargo, un marco espacial general que forma una «máquina de aprendizaje» enraizada en diferentes tipos de espacios e instituciones (del micro local al regional), de trayectorias históricas locales y de marco de integración (Faludi, 2010) y no exentas de tensiones y de conflictos.

Tabla 1. INTERREG. Tipos de cooperación y escala

\begin{tabular}{llll}
\hline & INTERREG A & INTERREG B & INTERREG C \\
\hline Referente espacial & Proximidad & Continuidad & Red \\
\hline $\begin{array}{l}\text { Escala/tipo de } \\
\text { cooperación }\end{array}$ & $\begin{array}{l}\text { Transfronteriza en sen- } \\
\text { tido estricto, desarrollo } \\
\text { regional integrado }\end{array}$ & $\begin{array}{l}\text { Transnacional, grandes } \\
\text { grupos de regiones }\end{array}$ & $\begin{array}{l}\text { Interregional, intercam- } \\
\text { bios y organización en } \\
\text { red }\end{array}$ \\
\hline
\end{tabular}

Fuente: elaboración propia. 


\section{Los espacios transfronterizos y la cooperación transfronteriza a lo largo del proceso de construcción de la Unión Europea}

Como se apunta en la introducción, las relaciones transfronterizas en Europa en los últimos setenta años —entiéndase en el ámbito de la UE- han sido motivo de una larga y profunda reflexión. Este hecho entra dentro de toda lógica, puesto que la frontera, como símbolo e instrumento de la soberanía estatal tendría que ser una de las "víctimas» de un proceso político, económico y cultural (Keating, 1998; Nogué y Vicente, 2001; Castells, 1997) de integración supraestatal como es la UE. Así, la eliminación o matización de la frontera la transforma en lo contrario: símbolo e instrumento de la nueva entidad que se alimenta, precisamente y en gran medida, de la transferencia de soberanía de los estados.

Partiendo de esta premisa/constatación a la que hacemos referencia reiteradamente, el análisis de estas relaciones requiere mucha más precisión. Simplemente tomando las palabras como punto de arranque, cuando se refieren a relaciones y a transfronterizas, ¿de qué se está hablando?: ¿de competencia?; ¿de cooperación?; ¿¿de políticas económicas, culturales...?; ¿institucionales y regladas o civiles e informales?; ¿a qué escala: estatal, regional o local?; ¡en qué momento del proceso de construcción de la UE?

Si la perspectiva es esta última, la temporal, el análisis de estas relaciones ya es de por sí complejo, puesto que como mínimo su evolución depende de dos factores dominantes: el de la propia y compleja historia de la construcción europea (con sus vaivenes, ampliaciones...) y el de la evolución de las teorías, métodos e instrumentos de las políticas transfronterizas y, más en general, territoriales de la UE.

Con estos marcos, son bastantes los autores que han propuesto una periodización de las relaciones transfronterizas europeas desde los inicios en los años cincuenta hasta el presente. Existe un notable consenso en identificar tres etapas que cubren casi cincuenta años de historia, de la segunda mitad del siglo XX. Por ejemplo, De Sousa (2012) u Oliveras, Durà y Perkmann (2010) proponen tres etapas claras y estos últimos, como se ha dicho al inicio, una cuarta en ciernes que responden a hitos históricos bien definidos. A grandes trazos, las etapas son las siguientes.

\subsection{0-1980: cooperación transfronteriza sin debilitar fronteras}

Una primera etapa, como se ha dicho, transcurre desde finales de los años cincuenta hasta $1980^{1}$. Esta se caracteriza, por un lado, por unas relaciones en gran medida marcadas por las lógicas estatales — acuerdos entre gobiernos—;

1. Para interpretar en su justa medida los pasos dados en este período, no hay que olvidar que en cuanto a la actual UE se están refiriendo a un territorio compuesto en su inicio por seis estados y en su final a doce y el Consejo de Europa estaba formado inicialmente por doce estados; es decir, a un sentido muy restringido tanto territorial como conceptualmente de Europa: la aliada o modelada bajo el Plan Marshall, la democrática, la liberal... 
únicamente el Consejo de Europa promueve relaciones más territorializadas como las Comunidades de Trabajo y ampara iniciativas como las primeras Eurorregiones o la Asociación Europea de Regiones Fronterizas. Y, por otro lado, se empiezan a establecer relaciones entre municipios más bien informales, de buena voluntad y de poca trascendencia política práctica pero de alto valor simbólico en algunos casos. Es decir, son un tipo de relaciones que no alteran la existencia de la frontera y que no afectan a la soberanía de los estados. Ello da pie, por el momento, a un mayor protagonismo del Consejo de Europa más que a las Comunidades Europeas, a pesar de que estas — en 1975 - crean los fondos de cooperación regional, los FEDER, que tanta importancia adquirirán en décadas posteriores (Medina, 2017).

\subsection{0-1990. Hacia una Unión Europea: las fronteras como realidades a superar}

La segunda etapa se inicia en 1980, con el denominado Convenio de Madrid, promovido de nuevo por el Consejo de Europa bajo el título de Convenio marco europeo de cooperación transfronteriza entre autoridades y comunidades territoriales. Como puede leerse desde el mismo título, la finalidad de dicho acuerdo da el papel protagonista a las instituciones subestatales, e insinúa un paso cualitativo relevante en cuanto a la idea de unificación e integración europea que supere el carácter estrictamente económico inicial y que irá tomando forma, precisamente, en esta década.

Los años ochenta, en realidad, no muestran demasiados frutos de dichos acuerdos - a pesar de que en áreas donde la cooperación fue pionera (Benelux...) se fuera consolidando-. Sin embargo, estos años son decisivos para Europa, sus regiones y municipios y sus fronteras. En efecto, en buena parte debido, en primer lugar, a un marco geopolítico mundial de nuevo fuertemente tensionado por la Guerra Fría y por grandes transformaciones económicas y tecnológicas, se impulsa la idea de una UE como entidad política supraestatal con un cierto carácter de confederación y como determinante actriz global (Castells, 1997; Vicente, 1993).

Por otro lado, se produce también la ampliación hacia el sur, con la entrada de Grecia (1981), España y Portugal (ambos en 1986), que da pie a reequilibrios geopolíticos y pone más en evidencia los desequilibrios regionales internos. Y en tercer lugar y a otro nivel, esta década es la de la eclosión de las teorías de desarrollo económico regional y local —industrialización endógena, distritos industriales, sistemas territoriales locales...-, de los modelos bottom$u p$, de las ciudades como nuevos motores de crecimiento..., y alternativos a los grandes conglomerados industriales públicos o privados y a las lógicas de los "polos de desarrollo» tan característicos de las políticas estatalistas de las décadas anteriores (Dematteis y Governa, 2005).

Además, en el marco institucional, unos cuantos países miembros de la entonces denominada Comunidad Económica Europea (CEE) — como Italia, Francia o España - se abren a modelos de administración más o menos des- 
centralizada, que dan cobertura legal y competencial a las políticas de desarrollo de las regiones y los municipios y a sus acciones, consecuencia de las teorías antes referidas, que multiplican las eurorregiones, las redes de cooperación, las áreas funcionales (Paasi, 2009).

\subsection{0-2008. De la Europa cohesionada a la Europa competitiva}

En definitiva, si se sigue con la periodización de Oliveras, Durà y Perkmann (2010), esta segunda etapa parece fundamental en relación a los espacios transfronterizos, no tanto por ella misma como por las condiciones de partida de una tercera fase, esta sí, determinante: la que se inicia en 1990 y se desarrolla durante la siguiente década y media. Así, el Acta Única Europea (1986) lleva a un inigualado avance del proceso de integración, muy relevante por su impacto en los espacios fronterizos. Conduce también a la «nueva» UE surgida del tratado de Maastricht (1992), a la aplicación en 1995 de los acuerdos de Schengen para la libre circulación, a la moneda única (2002)... Y pone en marcha programas y mecanismos de cooperación específicos para estos espacios transfronterizos, entre los que destacan las Agrupaciones Europeas de Interés Económico (creadas en 1985) y, sobre todo, la iniciativa INTERREG A (primera convocatoria de 1990), como eje específico de los FEDER antes mencionados.

Centrando la evolución de las políticas en INTERREG, este programa en sus ya cinco convocatorias se configura como uno de los ejes característicos de las actuaciones de la UE, considerando además el hecho de que las sucesivas ampliaciones, especialmente hacia el Este, ponen todavía más en evidencia las disparidades sociales entre los diferentes estados y, más concretamente entre regiones, clamorosas en casos de territorios limítrofes fronterizos. Por otro lado, otras iniciativas comunitarias también colocan los espacios transfronterizos en el centro de las políticas europeas, muy especialmente las infraestructurales por medio de la Red Transeuropea de Transporte (TEN-T), que se va definiendo, de nuevo, desde inicios de los noventa ${ }^{2}$, a menudo en medio de grandes debates ambientales y geopolíticos.

El desarrollo de las convocatorias de INTERREG entre 1990 y 2014 supone un incremento importante de los fondos aportados; pero, además, pone necesariamente de manifiesto algunos aspectos para profundizar y otros para corregir en el futuro. Estos últimos son de especial interés, puesto que dan lugar a iniciativas relevantes para la cooperación transfronteriza y, para algunos autores (Oliveras, Durà y Perkmann, 2010), significa de hecho la entrada en una cuarta fase de las políticas. En este sentido, dos aspectos fundamentales que se visualizan son, por un lado, la evidencia de que una proporción relevante de proyectos presentados tienen como único objetivo la captación de recursos sin ningún tipo de sentido real de cooperación transfronteriza (Scott, 2016; van Houtum et al., 2016; Medina, 2017). Por otro lado, emergen también las

2. <http://ec.europa.eu/transport/infrastructure/tentec/tentec-portal/site/index_en.htm>. 
múltiples dificultades de cooperación entre territorios pertenecientes a estados distintos; es decir, con modelos de administración, regímenes jurídicos... diferentes que impiden o ralentizan extraordinariamente proyectos en algunos casos de gran interés y/o necesidad. Como consecuencia de todo ello, en 2006 se crea la figura jurídica de las Agrupaciones Europeas de Cooperación Territorial (AECT), con el objetivo de agilizar los instrumentos de gestión de los proyectos, y que en principio tendrán una gran incidencia en las nuevas convocatorias de INTERREG de 2007 y de 2014.

\subsection{8-: La Unión Europea en una encrucijada, y sus fronteras también}

Como hemos dicho, todo esto da pie a autores como Oliveras, Durà y Perkmann (2010) o Berzi (2017b) a hablar de la cuarta fase de la cooperación transfronteriza marcada por los objetivos de 2007. Sin embargo, bajo nuestro punto de vista, para entender mejor este último período es necesario ampliar el zoom y situar las nuevas programaciones INTERREG y sus nuevos instrumentos en un contexto de profundos cambios conceptuales y de coyuntura. Una perspectiva más amplia que nos lleva a sostener que lo que caracteriza esta etapa no serían exactamente las innovaciones pretendidas con las AECT, la búsqueda de la competitividad, etc., sino más bien las consecuencias de transformaciones más amplias y profundas y en cierta medida contrarias a dichas innovaciones.

En efecto, la primera década del siglo supone respecto al proyecto europeo cambios de enfoque relevantes en cierta manera, una corrección de rumbo respecto al modelo ideado en los años ochenta-noventa que, a su vez, responden a cambios todavía más amplios de escala global. Cabe recordar que aquel modelo surgió como «alternativa» a un mundo geopolíticamente bipolar; impulsado por líderes e ideologías de fuerte definición social (Castells, 1998; Vicente, 1993). En cambio, los últimos veinte años han venido marcados —además de por el derrumbe del bloque comunista, la reunificación alemana y sus consecuencias inmediatas - por nuevas incertidumbres y tensiones geopolíticas; por la ampliación de la Unión y el correspondiente movimiento de centros de gravedad; por la emergencia de China como potencia mundial; por una globalización regida en términos ideológicos por el liberalismo radical en cuanto a la movilidad de capitales...

De hecho, durante esta primera década de siglo XXI ya se modificaron algunos pilares del proyecto europeo que denotaban el cambio, en algún aspecto paradójico, hacia una mayor liberalización del funcionamiento y de las políticas de la Unión (con el Banco Central Europeo como garante de las mismas), acompañadas por una limitación de las competencias de las instituciones comunitarias en favor de las soberanías estatales. Esto es lo que se deduce del fracaso del Tratado de Constitución Europea de 2004 (cuando, después de un duro debate durante su redacción, los Países Bajos y Francia votaron en contra del mismo en sus respectivos referéndums y se bloqueó el proceso de unificación) o del Tratado posterior de Lisboa de 2007 que, entre otras cosas, revisaba los acuerdos de Maastricht (y su posterior desarrollo en Ámsterdam en 1997). 
Y para acabar de definir el marco de la situación actual de los espacios fronterizos y la cooperación entre ellos solo queda mencionar el estallido de la crisis de 2008 y su impacto y la confirmación de los riesgos terroristas (con un Mediterráneo en ebullición). Es decir, no tan solo se han redefinido modelos sociales y sus políticas correspondientes, sino que también se han revertido pasos dados hacia la integración, empezando por la libre circulación de personas (restricciones en los acuerdos de Schengen en base a amenazas terroristas o a la acogida de refugiados) y acabando con el reforzamiento de las opciones políticas contrarias a la UE y a las políticas de integración en general y favorables al reforzamiento de la soberanía de los estados hasta llegar al extremo del Brexit, la salida del Reino Unido de la Unión. Es decir, las fronteras en la última década más que tender a difuminarse han tendido a resurgir, sumiendo a los espacios fronterizos, tanto interiores como exteriores, en un momento claramente paradójico: de mayores posibilidades y necesidades de cooperación y, simultáneamente, de renacimiento de su rol como límite, filtro - como siempre, lo ha hecho seleccionando qué puede y qué no puede pasar-y separación.

En resumen, una cuarta etapa en la que la UE se encuentra en una nueva encrucijada y, como siempre ha sucedido, los espacios transfronterizos resultan afectados, colocados en una tensión entre las dinámicas simultáneas de desfronterización y refronterización que añaden complejidad a su comprensión y gestión.

\section{4. ¿De la cohesión territorial a los espacios blandos?}

Desde la perspectiva de la desfronterización y refronterización se abre, por lo tanto, un cuarto período con la implementación de la política de integración y cohesión territorial de la UE a finales de la década de 2000. Este periodo se enmarca en un contexto de reflexión territorial en el que una parte significativa de la literatura científica analiza los cambios en curso en base a la aparición de espacios blandos (Faludi, 2010; Allmendinger y Haughton, 2009; Allmendinger et al., 2015; Haughton y Allmendinger, 1998; Haughton et al., 2010). Al igual que lo que se ha observado en la planificación urbana y regional —que conduce a nuevas formas de gobernanza territorial de áreas más difusas y con los límites cambiantes que se adaptan a los proyectos y actores implicados-, las áreas transfronterizas constituyen a su vez una categoría de espacios blandos que se suman a los espacios tradicionales, estandarizados, gestionados por los estados (espacios duros).

Para Faludi (2013), la estrategia macrorregional formalizada en la política de integración de la UE y la cohesión territorial y promovida en varios informes europeos («Libro Verde de la cohesión territorial», UE, 2008; «Informe Barca», 2009) alienta el desarrollo de otras formas de territorialidad que cumplan los objetivos del desarrollo territorial integrado para una Europa coherente. Tomando el ejemplo de la política de integración europea llevada a cabo en algunas Eurorregiones a través de los programas INTERREG (región del Mar Báltico y región del Danubio), varios autores (Dühr, 2011; Faludi, 2010) han demostrado que la UE facilita desarrollo de macrorregiones con límites difusos y cambiantes, débilmente institucionalizados, asociando diferentes territoria- 
lidades y actores para la gobernanza multinivel. Estas macrorregiones toman la forma de espacios blandos que combinan diferentes escalas de planificación y gobernanza, con unos límites que flexibilizan los estrictamente administrativos de los estados y regiones. Así, la política de integración territorial de la UE llevaría a producir un conjunto complejo y evolutivo de las tensiones y nuevos espacios de gobierno que se combinan a diferentes escalas en las zonas fronterizas (de lo local a lo regional) (Allmendinger y Chilla, 2014). En algunos ejemplos — regiones del Báltico o el Danubio o Neisse-Nisa-Nysa (Allmendinger y Chilla, 2014) —, la complejidad de muchas territorialidades que se superponen a diferentes escalas (de estados, regiones y ciudades) y las tensiones asociadas con ellas (en relación con la partición entre los estados y unidades administrativas) se superan gracias a la creación de espacios a medida (bespoke spaces) (Faludi, 2013). Los soft spaces, como espacios ad hoc, permiten colaboraciones transfronterizas en diversos campos (educación, salud, transporte, medio ambiente, economía...), con delimitaciones borrosas y variables en función de los temas y actores.

En estos casos, las políticas de cooperación transfronteriza constituyen una forma de «territorialidad aspiracional» que difiere de la «territorialidad dura» (la de los estados), porque no se basa en normas políticas y territorios delimitados, sino en normas aspiracionales y objetivos políticos, compartidos por los actores en espacios con geografía variable y límites difusos. Para Faludi (2013), los espacios flexibles reflejan nuevas formas de territorialidad producidas en el contexto de la gobernanza transfronteriza multinivel, donde el alcance de las fronteras es limitado. Estos espacios blandos se caracterizan por acuerdos institucionales a diferentes escalas y donde las formas nuevas de gobernanza, a menudo informales, se superponen con las formas tradicionales de gobierno (incluidas las de los estados que continúan influyendo). Según estos autores, el concepto de espacios blandos permite comprender mejor la complejidad espacial, política y escalar (gobernanza multinivel, multiplicidad de actores) de los espacios transfronterizos mediante la asociación en el análisis científico de enfoques territoriales y relacionales. Los espacios de cooperación, blandos, asocian una constelación de actores que comparten una misma estrategia espacial. Una nueva geografía de fronteras y áreas fronterizas emergería en la forma de nuevos conjuntos de territorios, redes de actores, autoridades e imaginarios territoriales. Por otra parte, y tan solo lo mencionamos sin entrar a fondo, los espacios blandos también ofrecen oportunidades a áreas transfronterizas que se asientan en territorios divididos por una frontera administrativa pero que comparten elementos identitarios de carácter cultural, histórico o, incluso, político.

Un buen ejemplo de ello es el que se deduce del análisis de los proyectos de cooperación transfronteriza entre España y Francia en el marco de los programas INTERREG III y IV, que a nuestro entender corroboran las dos propuestas centrales de este artículo: que los espacios transfronterizos pueden clasificarse como soft spaces y que en la última década una cierta refronterización genera nuevas rigideces en ellos. Se trata de un análisis (Castañer y Feliu, 2012; Feliu et al., 2013) de más de 200 proyectos para los períodos 2000-2006 y 2007-2013, 
Figuras 1 y 2. Número de proyectos por actores territoriales (NUTS II, III, IV y V) para la frontera franco-española en INTERREG III y IV (1999-2007 Y 2008-2014)
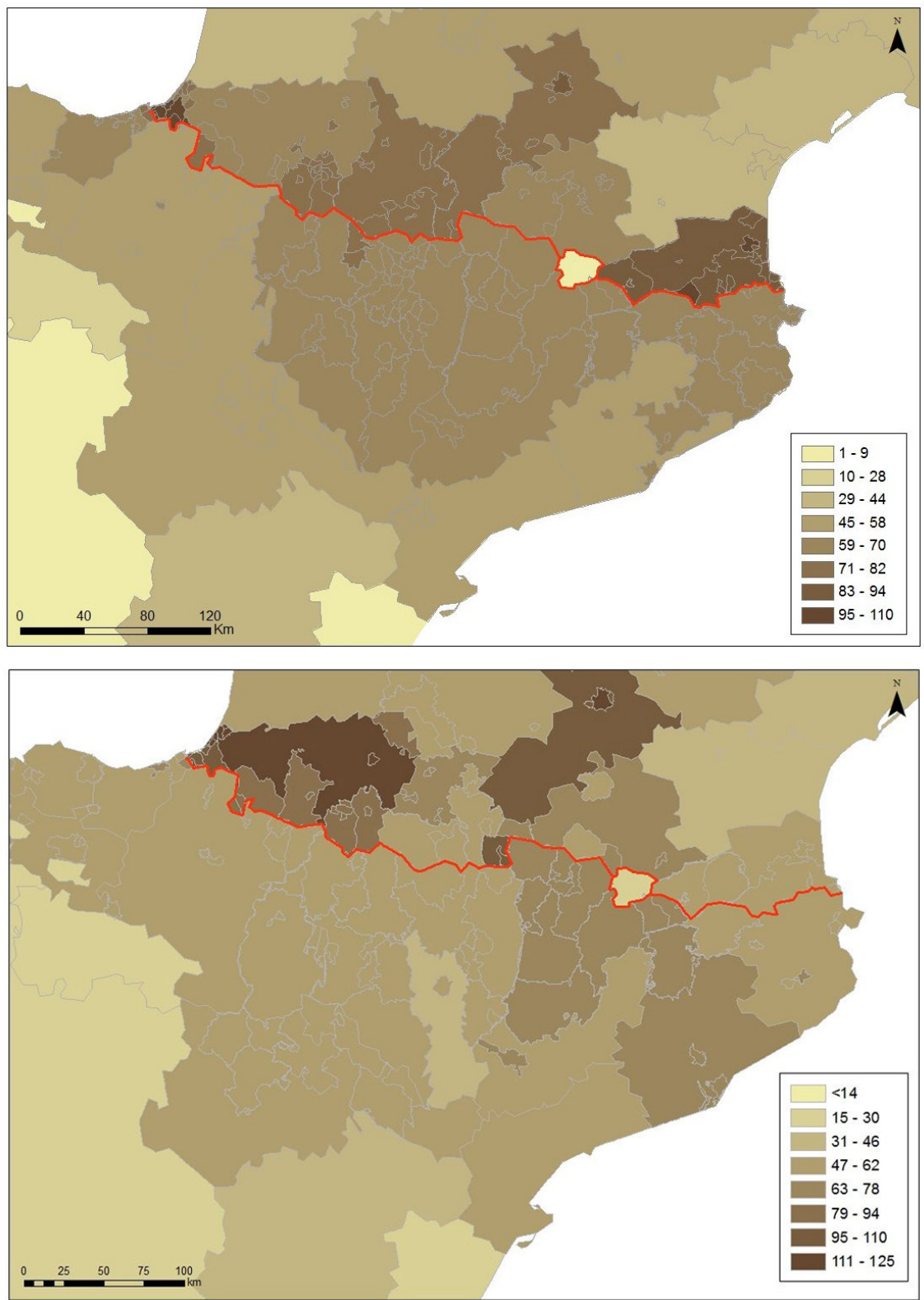

Fuente: elaboración propia, APTA-UdG (Matteo Berzi y Anna Céspedes). 
es decir, en coincidencia, entre una convocatoria y otra, con el cambio de ciclo en las políticas de cooperación transfronteriza que apuntábamos anteriormente. Lo que sobresale de los datos es, por un lado, el incremento de recursos — $181 \mathrm{M}$ de $€$ a $245 \mathrm{M}$ de $€$ — y la disminución de proyectos, un indicador a nuestro entender tanto de la apuesta de la UE por la cooperación como de un mayor cuidado en la concesión de financiación, atendiendo más a criterios de eficiencia - como se ha dicho, cuestionada a menudo a partir de experiencias de uso irregular o meramente financiero de los recursos- y competitividad, de acuerdo con las directrices de Lisboa. Y como hipótesis, seguramente también las políticas de austeridad aplicadas por los estados pueden explicar el descenso en el número de proyectos que se detecta, puesto que como se sabe los proyectos INTERREG exigen la cofinanciación. Reforzando esta hipótesis para el caso español, sin duda han impactado en el descenso de proyectos las políticas restrictivas del gobierno central relativas a los consorcios y otros tipos de instituciones supramunicipales y/o de cooperación público-privada, en el marco de las políticas de austeridad que desembocan en la Ley 27/2013, de 27 de diciembre, de racionalización y sostenibilidad de la Administración Local.

Por otro lado, la simple visión de los mapas pone de manifiesto primeramente el peso de las entidades locales en sus diversas tipologías - municipios, comarcas, communautées d'agglomération, pays- e instituciones no políticas - consorcios, universidades o cámaras de comercio-. Estas entidades locales, representadas por los NUTS IV y V (LAU) en el estudio mencionado, suponen para los dos períodos un porcentaje superior al $80 \%$ del total de actores. Pero, más relevante para nuestra propuesta, en la comparación de las figuras 1 y 2, es observar que la delimitación del espacio transfronterizo es claramente móvil e imprecisa en sus estructuraciones internas para la cooperación. O mejor dicho: si bien las normativas de INTERREG prefiguran un marco territorial preciso las regiones que pueden acceder a sus recursos-, esta delimitación en la práctica sirve «únicamente» como un marco de máximos dentro del cual distintos tipos de entidades y de ámbitos se articulan de una manera flexible de un período a otro; unos «espacios blandos» que se confrontan con un imaginario, tradición y práctica de la frontera como un dato geográfico exactamente de características contrarias a estas: preciso e inmóvil (e, incluso, solo alterable como consecuencia de conflictos). Es decir, refleja que el espacio transfronterizo es fundamentalmente consecuencia de los proyectos transfronterizos, y no viceversa.

\section{Conclusión: los espacios transfronterizos como espacios blandos y la crisis de la Unión Europea: entre la desfronterización y la refronterización}

Más que nunca, las zonas transfronterizas europeas son hoy en día objeto de fuertes tensiones entre los movimientos de reescalamiento y desfronterización y movimientos inversos de refronterización. Los primeros impulsados por los intereses de la globalización, la cooperación internacional, la política de cohesión territorial de la UE y por dinámicas de carácter local; y los segundos 
dirigidos al reforzamiento de los principios estatales de la soberanía o a cierres temporales o permanentes de fronteras.

En efecto, las diversas crisis que se han superpuesto y que han afectado a la UE desde 2008 tienen múltiples efectos en las áreas de cooperación transfronteriza. La crisis financiera está llevando a muchos estados y regiones a reducir los presupuestos de cooperación transfronteriza. La crisis política de la desconfianza popular hacia la UE es una constante en la vida política de varios estados (como lo demuestra el ejemplo paroxístico del Brexit). La crisis de los refugiados y la incapacidad de la UE para proporcionar una respuesta coordinada han llevado a varios países de Europa Central a cerrar sus fronteras. En cuanto a la crisis de seguridad, autoriza, en nombre de la lucha contra el terrorismo, a fortalecer los controles fronterizos y limitar de facto las movilidades y la cooperación. En definitiva, después de casi más de tres décadas de discursos y acciones tendentes a la matización de los efectos separadores de las fronteras, asistimos a discursos, decisiones y acciones en sentido contrario, en muchos casos con el aval de la ciudadanía expresado en votos.

Todo ello significa un manifiesto cambio de tendencia respecto al período final del siglo XX e inicial del siglo XXI, durante el cual la UE respondía a un impulso explícito de cooperación y cohesión, identificado como múltiples procesos de desfronterización (Faludi, 2009). De él surgieron los espacios transfronterizos caracterizados por la difuminación de la frontera entendida como separación y por la configuración de áreas de proyecto surgidas desde la escala local y/o regional, sin delimitación previa, sin rigideces preconcebidas, como espacios blandos. Esta característica daba a los espacios transfronterizos una doble importancia: eran áreas emblemáticas de un proceso de reforma económica, geopolítica..., de magnitud global — la construcción de la UE—; y simultáneamente permitían experimentaciones de gobierno y gobernanza innovadoras con efectos claros de desarrollo local. En resumen, la última etapa de la cooperación transfronteriza se caracteriza por una convivencia tensa entre inercias características de espacios blandos y tentaciones de territorialidad «dura».

$\mathrm{Si}$, como decíamos al inicio, el espacio transfronterizo es un indicador de la «salud» y la confianza del ideal europeísta, entonces el momento actual es indudablemente de profunda incertidumbre.

\section{Referencias bibliográficas}

AGNEW, John (1994). «The territorial trap: the geographical assumptions of international relations theory». Review of International Political Economy, 1 (1), 53-80. <http://doi.org./10.1080/09692299408434268>

Allmendinger, Phil; Haughton, Graham; Knieling, Jörg y Othengrafen, Frank (eds.) (2015). Soft Spaces in Europe, Re-negotiating governance, boundaries; borders. Londres: Routledge.

Allmendinger, Phil y Chilla, Tobias (2014). «Europeanizing territoriality, towards soft spaces?». Environment; Planning A, 46, 2703-2717.

$<$ https://doi.org/10.1068/a130037p > 
Allmendinger, Phil y Haughton, Graham (2009). «Soft spaces, fuzzy boundaries; metagovernance: the new spatial planning in the Thames Gateway». Environment; Planning A, 41, 617-633. $<$ https://doi.org/10.1068/a40208>

Amilhat-Szary, Anne-Laure y Fourny, Marie-Christine (ed.) (2006). Après les frontières, avec la frontière. Nouvelles dynamiques transfrontalières en Europe. La Tour d'Aigues: L'Aube, 169.

BAUDER, Harald (2011). "Toward a critical geography of the border: engaging the dialectic of practice; meaning». Annals of the Association of the American Geographers, 101 (5), 1126-1139. <https://doi.org/10.1080/00045608.2011.577356>

BERZI, Matteo (2017a). «The cross-border reterritorialization concept revisited: the territorialist approach applied to the case of Cerdanya on the French-Spanish border». European Planning Studies, 25 (9), 1575-1596. <https://doi.org/10.1080/09654313.2017.1321622>

- (2017b). «La vía transfronteriza del desarrollo territorial endógeno. Reflexiones teóricas y estudios empíricos en los Pirineos y los Alpes». Universitat Autònoma de Barcelona, tesis doctoral.

BLATTER, Joachim (2004). «From 'spaces of place' to 'spaces of flows'? Territorial; functional governance in cross-border regions in Europe; North America». International Journal of Urban; Regional Research, 28 (3) 530-548. $<$ https://doi.org/10.1111/j.0309-1317.2004.00534.x>

BRENNER, Neil (1999). "Globalization as reterritorialization: The Re-scaling of Urban Governance in the European Union». Urban Studies, 36 (3), 431-451. <https://doi.org/10.1080\%2F0042098993466>

BRUNET-JAILlY, Emmanuel (2005). «Theorizing borders: an interdisciplinary perspective». Geopolitics, 10 (4), 633-649.

CASTAÑER, Margarida y FELIU, Jaume (2012). «L'Eurodistricte català transfronterer. Un espai emergent sense marc administratiu». Treballs de la Societat Catalana de Geografia, 74, 41-58.

CASTELLS, Manuel (1997). L'era de la informació. Vol. II: El poder de la identitat. Barcelona: UOC, 2003.

- (1998). L'era de la informació. Vol. III: Canvi de mil.lenni. Barcelona: UOC, 2003.

CONSEJO DE EUROPA (1980). Convención sobre cooperación transfronteriza entre comunidades territoriales. <http://www.interior.gob.es/web/servicios-al-ciudadano/normativa/acuerdos-y-convenios/convenio-marco-de-21-de-mayo-de-1980>. [Consulta: 28 de octubre de 2017]

DE SOUSA, Luis (2012). «Understanding European crossborder cooperation: a framework for analysis». Journal of European Integration, iFirts Article, 1-19. <https://doi.org/10.1080/07036337.2012.711827>

Dematteis, Giuseppe y Governa, Francesca (2005). Territorialità, sviluppo locale, sostenibilità: il modello Slot. Milán: Franco Angeli.

DüHR, Stefanie (2011). «Baltic sea, Danube and macro-regional strategies: a model for transnational cooperation in the UE?». Radboud Repository. Nijmegen: Radboud University Nijmegen.

Evrard, Estelle, (2013). «L'espace frontalier, région en construction». Belgeo, 1. <https://doi.org/10.4000/belgeo.10573> 
FALUDI, Andreas (2009). "A turning point in the development of European spatial planning? The 'Territorial Agenda of the European Union'; the 'First Action Programme'». Progress in Planning, 71 (1), 1-42. <https://doi.org/10.1016/j.progress.2008.09.001>

- (2010). «Beyond Lisbon: soft European spatial planning». DISP, 182, 3, 14-24. Geopolitics, 19 (3), 587-608. <https://doi.org/10.1080/02513625.2010.10557098>

- (2013). "Territorial cohesion, territorialism, territoriality; soft planning: a critical review». Environment; Planning A, 45, 1302-1317. $<$ https://doi.org/10.1068/a45299>

Feliu, Jaume; BerZi, Matteo; Vicente, Joan; CAStañer, Margarida y Llussà, Rafel (2013). «Análisis de los proyectos y actores transfronterizos España-Francia en el período 2007-2013». Geographicalia, 63-64, 75-93.

GUALINI, Enrico (2003). "Cross-border governance: inventing regions in a transnational multi-level polity». dis $P, 39$ (152), 43-53. <https://doi.org/10.1080/02513625.2003.10556833>

Hamez, Gregory (2013). "Vers un modèle multiscalaire des territoires frontaliers intérieurs à l'Union européenne». Belgeo, 1. <https://doi.org/10.4000/belgeo.10558>

HARGUINDÉGUY, Jean Baptiste y HAYWARD, Katy (2014). «The institutionalization of the European internal cross-border co-operation policy: a first appraisal». European Planning Studies, 22 (1), 184-203. <https://doi.org/10.1080/09654313.2012.741571>

Haughton, Graham y Allmendinger, Phil (1998). «The soft spaces of local economic development», Local Economy, 23, 138-148.

Haughton, Graham; Allmendinger, Phil; Counsell, David y Vigar, Geoff (2010). The new spatial planning: territorial management with soft spaces; fuzzy boundaries. Londres: Routledge.

Herzog, Lawrence y SoHN, Christophe (2015). «The co-mingling of bordering dynamics in the San Diego-Tijuana crossborder metropolis», Working Paper, 2016-1, Luxembourg Institute of Socio-economic Research, 21 pág.

KAPLAN, David y HäKLI, Jouni (2002). Boundaries; place: European borderlands in geographical context. Londres: Rowman \& Littlefield.

KeATING, Michael (1998). The new regionalism in Western Europe. Territorial restructuring; political change. Northhampton: Edward Elgar Pub.

MEDINA, Eusebio (2017). «Marco jurídico y principales instrumentos de la cooperación transfronteriza institucional en Europa». Investigaciones Regionales, 37, 189-206.

NoguÉ, Joan y Vicente, Joan (2001). Geopolitica, identidad y globalización. Barcelona: Ariel.

Oliveras, Xavier; DurÀ, Antoni y Perkmann, Markus (2010). «Las regiones transfronterizas: balance de la regionalización de la cooperación transfronteriza en Europa (1958-2007)». Documents d'Anàlisi Geogràfica, 56 (1), 21-39.

PAASI, Ansi, (2009). "Bounded spaces in a "borderless world": border studies, power; the anatomy of territory». Journal of Power, 2 (2), 213-234. <https://doi.org/10.1080/17540290903064275>

PERKMANN, Markus (2003). «Cross-border regions in Europe significance; drivers of regional cross-border cooperation», European Urban; Regional Studies, 10 (2), 153-171. <https://doi.org/10.1177/0969776403010002004> 
- (2005). «The emergence; governance of Euroregions: the case of the EUREGIO on the Dutch-German border", Conference Papers; Presentations (Mechanical, Electrical; Manufacturing Engineering). <https://dspace.lboro.ac.uk/2134/743>.

PIERMAY, Jean-Luc (2009). "La frontière, un outil de projection au monde. Les mutations de Tanger (Maroc)». Espaces et sociétés, 138 (3), 69-83. <https://doi.org/10.3917/esp.138.0069>

Rumley, Dennis y Minghi, Julian (eds.) (1991). The geography of border landscapes. Londres: Routledge.

ScotT, James Wesley (2005). «The EU and 'Wider Europe': toward an alternative geopolitics of regional cooperation?». Geopolitics, 10, 429-454. <https://doi.org/10.1080/14650040591003471>

- (2016). «Rebordering Central Europe: observations on cohesion; cross-border cooperation». Cross-border Review Yearbook. European Institute on Cross-border Studies 2016. <http://institute.cesci-net.eu/en/crossborder-review-2016>

SoHn, Christophe (2014a). «The Border as a Resource in the Global Urban Space: A Contribution to the Cross-Border Metropolis Hypothesis». International Journal of Urban; Regional Research, 38 (5), 1697-1711. <https://doi.org/10.1111/1468-2427.12071>

- (2014b). «Modelling cross-border integration: the role of borders as a resource». Geopolitics, 19 (3), 587-608. <https://doi.org/10.1080/14650045.2014.913029>

Sohn, Christophe y STAmbOlic, Nora (2015). "The urban development of European border regions: a spatial typology». Europa Regional, 21 (4), 177-189.

UNIÓN EUROPEA (2008). Libro verde de la cohesión territorial. El camino a seguir. $<$ http://ec.europa.eu/regional_policy/sources/docgener/panorama/pdf/mag28/ mag28_es.pdf>

VAN HOUTUM, Henk (2010). «The mask of the border». En: Doris WASTL-WALTER. The Ashgate research companion to border studies. Ashgate, 11-33. $<$ https://doi.org/10.1002/9781118255223.ch23>

VAn Houtum, Henk; Laine, Jussi y ScotT, James Wesley (2016). «European crisis; its consequences for borders; cooperation». Cross-Border Review Yearbook. European Institute on Cross-border Studies, 135-152.

VICENTE, Joan (1993). «Construcción, deconstrucción y reconstrucción de Europa». Boletín de la Asociación de Geógrafos Españoles, 17, 167-179.

\section{Otra documentación consultada}

Europa 2020. Una estrategia para el crecimiento inteligente, sostenible e integrador. <http://eur-lex.europa.eu/legal-content/ES/TXT/PDF/?uri=CELEX:52010DC2 020\&from=ES> [consulta: 23 de septiembre de 2017]

Informe Barca (2009). <http://ec.europa.eu/regional_policy/archive/policy/future/ barca_es.htm> [consulta: 15 de julio de 2017]

Keep.eu, estadística europea sobre programas transfronterizos <https://www.keep.eu/ keep/statistics $>$ [consulta: 25 de septiembre de 2017]

Interreg POCTEFA <https://www.poctefa.eu/> [consulta: 23 de septiembre de 2017] 